\title{
The Etiology of Pyogenic Vertebral Osteomyelitis and Evaluation of Biopsy Specimen Cultures in Hospitalized Patients
}

Masahiro Kamono $^{1 *}$, Yusuke Kabeya ${ }^{1}$, Eiseki Sohara ${ }^{1}$, Akiko Taoda ${ }^{1}$, Tomohiro Matsumoto ${ }^{2}$, Takahiko Mine ${ }^{2}$, Terumitsu Hasebe ${ }^{2}$, Akihiro Ueda ${ }^{1}$, Atsushi Takagi $^{1}$ and Megumu Higaki ${ }^{1}$

${ }^{1}$ Department of General Internal Medicine, Tokai University Hachioji Hospital, Tokai University School of Medicine, Japan

${ }^{2}$ Department of Radiology, Tokai University Hachioji Hospital, Tokai University School of Medicine, Japan

"Corresponding author: Masahiro Kamono, Department of General Internal Medicine, Tokai University Hachioji Hospital, Tokai University School of Medicine, Japan, Tel: +81-42-639-1111; Fax No: +81-42-639-1144; E-mail: kamono@tsc.u-tokai.ac.jp

Rec Date: September 08, 2017; Acc Date: October 09, 2017; Pub Date: October 12, 2017

Copyright: ( 2017 Kamono M, et al. This is an open-access article distributed under the terms of the Creative Commons Attribution License, which permits unrestricted use, distribution, and reproduction in any medium, provided the original author and source are credited.

\begin{abstract}
Identifying the causative pathogens in patients with pyogenic vertebral osteomyelitis is important for optimizing antibiotic therapy. Thus, we examined patient characteristics and investigated the efficacy of the combined use of blood culture and image-guided biopsy culture for identifying the causative pathogens of pyogenic vertebral osteomyelitis. A case series study was conducted in hospitalized patients who were diagnosed with pyogenic vertebral osteomyelitis using electronic medical records. Twenty-three patients who had undergone both blood culture and image-guided biopsy to determine the causative pathogens were identified. Detailed patient characteristics were identified, and the detection rate of a causative pathogen was $57 \%$ using either blood or biopsy specimen culture. By combining the results of each culture, the detection rate was increased to $83 \%$. We described the detailed characteristics of patients with pyogenic vertebral osteomyelitis from whom both blood and biopsy specimen cultures were obtained. We found that the combined use of blood culture and biopsy specimen culture is useful for enhancing the detection of causative pathogens in patients with pyogenic vertebral osteomyelitis.
\end{abstract}

Keywords: Pyogenic vertebral osteomyelitis; Patient characteristics; Blood culture; Biopsy specimen culture; Antibiotic exposure

\section{Abbreviations \\ PVO: Pyogenic Vertebral Osteomyelitis; MRI: Magnetic Resonance Imaging; CT: Computed Tomography; CRP: C-Reactive Protein; ESR: Erythrocyte Sedimentation Rate; MRSA: Methicillin- Resistant Staphylococcus aureus, IDSA: Infectious Diseases Society of America.}

\section{Introduction}

As society ages, the incidence of pyogenic vertebral osteomyelitis (PVO) has increased [1]. Elderly people are more likely to have degenerative spine disease, diabetes, or cancer, which are risk factors for PVO. Meanwhile, immunosuppressive therapies have become widely available, which could further accelerate the incidence of the disease. Although it is a serious disease, nonspecific symptoms such as back pain and fever and the infrequency of PVO often impede its diagnosis [2].

In the treatment of PVO, identifying the causative pathogen is important for optimizing antibiotic therapy. Blood culture is a gold standard for identifying the pathogens, which could facilitate the selection of appropriate antibiotics. However, in clinical practice, the detection rate of pathogens using blood culture is not high [3], and empirical antibiotic treatment without adequate culture may lead to unnecessary, prolonged broad-spectrum antibiotic use. An alternative method could be a direct approach to the affected lesions. At present, medical advances in imaging have improved the safety of approaching these lesions. In our hospital, we obtained both blood and specimen cultures via image-guided local biopsy and used the results to select appropriate antibiotics for patients with PVO. In the present study, we conducted a retrospective review of such patients to study the characteristics and investigate the usefulness of blood culture, imageguided biopsy, and their combined use.

\section{Patients and Methods}

We conducted a case series study of hospitalized patients with a diagnosis of PVO who were admitted to Tokai University Hachioji Hospital, a 450-bed general hospital located in a suburb of Tokyo, between April 2013 and November 2016.

The diagnosis procedure combination administrative database was used to identify all discharges using International Classification of Diseases, Tenth Revision (ICD-10) codes for infectious or unspecified osteomyelitis of vertebrae or intervertebral discs (M46.1, M46.2, M46.3, M46.5, M46.8, and M46.9) and unspecified discitis (M46.4). Discharges containing these ICD-10 codes were reviewed by one of the authors (M.K.) to determine whether they met the study definitions. This study was approved by the Tokai University Clinical Research Administration Office (17R-009).

Hospitalized patients who were older than 20 years at the time of admission were included. The diagnosis of PVO was made on the basis of clinical features, namely symptoms, radiographic findings, and elevated inflammatory markers. The symptoms were new or worsening back or neck pain, occasionally with fever. Radiographic changes on magnetic resonance imaging (MRI) included decreased signal intensity in the vertebral bodies and disc and loss of endplate definition on T1weighted images and increased disc signal intensity with or without increased vertebral body signal intensity on T2-weighted images. For patients who could not undergo MRI because of implanted cardiac 
Citation: Kamono M, Kabeya Y, Sohara E, Taoda A, Matsumoto T, Mine T, Haseb T, Ueda A, Takagi A and Higaki M (2017) The Etiology of Pyogenic Vertebral Osteomyelitis and Evaluation of Biopsy Specimen Cultures in Hospitalized Patients. J Med Microb Diagn 6: 261. doi:10.4172/2161-0703.1000261

Page 2 of 5

electrical devices or claustrophobia, computed tomography (CT) was employed to identify these findings. The MRI and CT findings were confirmed by radiologists. Inflammatory markers including leukocyte counts and C-reactive protein (CRP) levels with or without the erythrocyte sedimentation rate (ESR) were investigated at the time of admission.

The following information was collected from medical records: demographic and diagnostic imaging data, affected vertebral level, route of infection, risk factors, laboratory findings at the time of admission, methods of image-guided biopsy, microbiological pathogens, and history of antibiotic exposure at the time of obtaining cultures.

Regarding microbiologic examinations, two sets of blood cultures were obtained from all patients at the time of admission. In addition to obtaining blood cultures, patients subsequently underwent imageguided biopsy to obtain an infected vertebrae or disc specimen for culture and pathological examination. Image-guided biopsy was performed by interventional radiologists [4].

\section{Results}

\section{Patient characteristics}

According to the requirements, 23 patients who had undergone both blood culture and image-guided biopsy with PVO were included in the present study.

The characteristics of the patients are described in Table 1 . The prevalence of PVO was high in males and at the age of over 50 years.
The lumbar spine was the most common affected site. Regarding the route of infection, hematogenous spread, namely bacteremia, was the most common route, accounting for 13 cases (57\%) including one patient with infective endocarditis. One patient developed PVO due to direct inoculation caused by a myelographic procedure, and the other patient experienced contiguous spread from cellulitis caused by acupuncture therapy. The route of infection in the other 11 patients (48\%) was unknown. Concerning risk factors for PVO, degenerative spine disease and diabetes mellitus were notable. Regarding laboratory findings at the time of admission, the levels of ESR and CRP, and not leukocyte counts, were significantly elevated. Concerning the method of image-guided biopsy, 20 patients (87\%) underwent needle biopsy with CT guidance, and the remaining three patients (13\%), including one patient with a neurological deficit who underwent subsequent surgery, received biopsy via radioscopy. No patient had complications due to the biopsy procedures, and abscess drainage was subsequently performed in 15 patients. The median duration between the blood culture and subsequent image-guided biopsy was 4 days (2.5-13, interquartile range).

\section{Microbiological findings}

The results of blood culture and biopsy specimen culture are shown in Table 2. Among the 23 patients, blood culture and biopsy specimen culture were each positive in 13 patients $(57 \%)$. By combining the results of blood culture and biopsy specimen culture, the causative pathogens were successfully identified in 19 patients (83\%). On the contrary, pathogens were not identified in the remaining 4 patients, although they were not exposed to antibiotics previously.

\begin{tabular}{|c|c|c|c|}
\hline \multicolumn{4}{|c|}{ Total $(\mathrm{N}=\mathbf{2 3})$} \\
\hline \multicolumn{2}{|l|}{ Age, years } & \multirow{2}{*}{$\begin{array}{l}67 \\
20\end{array}$} & \multirow{2}{*}{$\begin{array}{l}(57.5-79) \\
(87)\end{array}$} \\
\hline Sex, n (\%) & Male & & \\
\hline \multirow[t]{2}{*}{ Diagnostic imaging, n (\%) } & MRI & 20 & (87) \\
\hline & CT & 3 & (13) \\
\hline \multirow[t]{3}{*}{ Vertebral level, n (\%) } & Cervical & 3 & (13) \\
\hline & Thoracic & 1 & (4) \\
\hline & Lumbar & 20 & (87) \\
\hline \multirow[t]{3}{*}{ Initial symptom, n (\%) } & New or worsening back and/or neck pain & 23 & $(100)$ \\
\hline & Fever & 12 & (52) \\
\hline & Neurological deficit & 1 & (4) \\
\hline \multirow[t]{4}{*}{ Mechanism of infection, n (\%) } & Hematogenous spread & 13 & $(57)$ \\
\hline & Direct inoculation & 1 & (4) \\
\hline & Contiguous spread & 1 & (4) \\
\hline & Unknown & 11 & (48) \\
\hline \multirow[t]{3}{*}{ Risk factors, n (\%) } & Degenerative spine disease & 16 & (70) \\
\hline & Diabetes mellitus & 8 & (35) \\
\hline & Corticosteroid therapy & 3 & (13) \\
\hline
\end{tabular}


Citation: $\quad$ Kamono M, Kabeya Y, Sohara E, Taoda A, Matsumoto T, Mine T, Haseb T, Ueda A, Takagi A and Higaki M (2017) The Etiology of Pyogenic Vertebral Osteomyelitis and Evaluation of Biopsy Specimen Cultures in Hospitalized Patients. J Med Microb Diagn 6: 261. doi:10.4172/2161-0703.1000261

Page 3 of 5

\begin{tabular}{|l|l|l|l|}
\hline \multirow{4}{*}{$\begin{array}{l}\text { Laboratory findings, } \\
\text { median (interquartile range) }\end{array}$} & Other immunocompromised state & 3 & $(13)$ \\
\cline { 2 - 4 } & Prior spinal surgery & 2 & $(9)$ \\
\cline { 2 - 4 } & Endocarditis & 1 & $(4)$ \\
\hline Method of biopsy, $\mathrm{n}(\%)$ & Leukocyte count & 8.5 & $(5.2-11.1)$ \\
\cline { 2 - 4 } & C-reactive protein & 8.6 & $(2.6-13.2)$ \\
\cline { 2 - 4 } & Erythrocyte sedimentation rate * & 76.5 & $(62.8-5.5)$ \\
\hline $\begin{array}{l}\text { Abbreviations: MRI: Magnetic Resonance Imaging; CT: Computed Tomography Erythrocyte sedimentation rate data were available for 14 patients who had undergone } \\
\text { ESR testing Data are } \mathrm{n}(\%) \text { or median (interquartile range) }\end{array}$ & Needle biopsy by CT guidance & 20 & $(87)$ \\
\cline { 2 - 4 } & Needle biopsy by radioscopy & 3 & $(13)$ \\
\hline
\end{tabular}

Table 1: Characteristics and clinical findings in patients who underwent both blood culture and image-guided biopsies.

\begin{tabular}{|c|c|c|c|c|}
\hline \multicolumn{5}{|c|}{ Blood culture } \\
\hline \multirow{3}{*}{$\begin{array}{l}\text { Biopsy } \\
\text { culture }\end{array}$} & \multirow[t]{2}{*}{ Positive } & Positive & Negative & Total \\
\hline & & $7(30)$ & $6(26)$ & $13(57)$ \\
\hline specimen & Negative & $6(26)$ & $4(17)$ & $10(43)$ \\
\hline & Total & $13(57)$ & $10(43)$ & $23(100)$ \\
\hline
\end{tabular}

Data are presented as the number of patients (\%).

Table 2: Results of blood culture and biopsy specimen culture.

Details concerning the identified causative pathogens and types of positive culture are described in Table 3. In the present study, in addition to Streptococcus species and Staphylococcus aureus, anaerobes were the common pathogens. Among the pathogens, Streptococcus species were identified in five patients $(22 \%)$, with the bacteria identified using both blood and biopsy specimen cultures in one patient (Streptococcus species), blood culture only in two patients (Streptococcus agalactiae and Enterococcus faecalis), and biopsy specimen culture only in two patients (Streptococcus salivarius and Streptococcus agalactiae). Staphylococcus aureus, including methicillin-resistant Staphylococcus aureus (MRSA) in two patients, was identified from both blood and biopsy specimen cultures in four patients who had paravertebral and/or iliopsoas abscesses. Anaerobes were identified in four patients (17\%) from either two blood cultures (Obligatory anaerobic non-spore-forming gram-positive bacillus) or two biopsy specimen cultures (Peptostreptococcus species and obligatory anaerobic non-spore-forming gram-positive bacillus).

\begin{tabular}{|c|c|c|c|c|}
\hline Organism & Total $(\mathrm{N}=19)$ & $\begin{array}{l}\text { Both blood and biopsy specimen } \\
\text { culture }\end{array}$ & Blood culture only & Biopsy specimen culture only \\
\hline Streptococcus species & 5 & 1 & 2 & 2 \\
\hline Staphylococcus aureus & 4 & 4 & - & - \\
\hline Anaerobe & 4 & - & 2 & 2 \\
\hline Klebsiella pneumoniae & 2 & 1 & 1 & - \\
\hline Other & 4 & 1 * & $1 \dagger$ & $2 \ddagger$ \\
\hline
\end{tabular}

Table 3: Pathogens and types of positive culture.

In 19 patients whose causative pathogens were identified, five patients had been exposed to antibiotics before blood cultures were obtained. Among them, three patients were exposed to antibiotics for more than 4 days. Although a patient with PVO caused by Staphylococcus aureus received an effective treatment, a causative pathogen was successfully identified using both blood and biopsy specimen cultures.

\section{Discussion}

The present study described the characteristics of patients with PVO in a Japanese suburban setting. Regarding the patients' characteristics, their median age, sex ratio, affected vertebral levels, risk factors, and laboratory findings were similar to those reported in past studies $[1,3,5,6]$. On the contrary, compared to an analysis of 14 case series concerning the clinical characteristics of patients with PVO [3], the prevalence of neurological deficits in the present study was extremely low ( $34 \%$ vs. $4 \%$ ). The short period between the onset and diagnosis or relatively fewer number of patients with Staphylococcus aureus 
infection in the present study might explain the difference, as described in the past study [7], although detailed analysis is necessary.

Pathogen detection rates were assessed for blood culture and biopsy specimen culture, and we found that the combined use of blood culture and biopsy specimen culture successfully increased the rate of pathogen detection from $57 \%$ to $83 \%$. Although the difference was not statistically tested because of the small number of cases, this improvement supports the usefulness of image-guided biopsy. The Infectious Diseases Society of America (IDSA) clinical practice guidelines recommend the use of image-guided biopsy when a microbiologic diagnosis has not been established by blood culture or serologic tests [8]. However, this strategy could delay the initiation of treatment. Although the microbiologic yield of image-guided biopsy is considered to range from $36 \%$ to $91 \%$ according to the literature [7,9-12], our concurrent biopsy specimen culture might have produced a high yield and provided additional information for $26 \%$ of patients in our study. The present study suggests that the concurrent use of blood culture and biopsy specimen culture can produce a high detection rate with an acceptable lead time for the start of the treatment. After diagnosis, all 23 patients received appropriate antibiotic treatment according to the IDSA clinical practice guidelines [8]. Although only two patients (9\%), one patient with MRSA bacteremia and paravertebral abscess who was receiving chemotherapy for urethral cancer and the other with no identified pathogen who had an intestinal fistula after the surgery for colon cancer, experienced a recurrence of PVO within 8 weeks after the initiation of treatment, both were successfully treated after the recurrence (data not shown).

Image-guided biopsy is considered useful for both distinguishing between infected and non-infected lesions via pathologic examination and identifying microorganisms such as Mycobacterium tuberculosis, Brucella species, and fungi, which are difficult to detect via blood culture and serologic examination [13]. In the present study, one patient had PVO caused by Mycobacterium tuberculosis. Although serological testing produced a false-negative result and the radiographic change was non-specific, the patient was successfully diagnosed via biopsy specimen culture. Moreover, biopsy specimen culture would be useful for diagnosis in cases of local infection such as direct inoculation. We could detect an anaerobe (obligatory anaerobic non-spore-forming gram-positive bacillus) in a patient with contiguous spread from cellulitis caused by acupuncture. Meanwhile, the negative biopsy specimen culture findings might have resulted from insufficient tissue inoculum, fastidious microorganisms, or the impact of antibiotic administration. In the four patients whose causative pathogens were unknown, histology revealed inflammatory changes in vertebrae, and all patients responded to subsequent antibiotic therapy, although the possibility of spontaneous resolution cannot be excluded. Moreover, an analysis of the four patients whose pathogens (i.e., Streptococcus salivarius, Streptococcus agalactiae, Peptostreptococcus species, or Corynebacterium species) were identified in only biopsy specimen cultures is needed.

As Staphylococcus aureus and Streptococcus species are considered to be dominant pathogens in PVO $[1,3,5]$, it is also interesting that anaerobes were identified in four patients (13\%) in the present study. It seems to be difficult to identify an anaerobe as a causative pathogen in PVO because the success in the identification of an anaerobe depends on the quality of culture samples, the mode of transport, and the condition of cultures. Furthermore, PVO caused by anaerobic infection tends to occur in the post-surgical site and polymicrobial condition
[14]. In the present study, only one patient underwent the operation for lumbar canal stenosis.

Although hematogenous spread is said to be dominant in PVO [15], only one case of infectious endocarditis caused by Streptococcus agalactiae was identified in this study. Further analysis is necessary to reveal the primary focus.

We also investigated the impact of previous antibiotic exposure on the results of the blood and biopsy specimen culture. According to the clinical practice guidelines, pausing antibiotic therapy for 1 or 2 weeks before obtaining biopsy specimen is considered reasonable when feasible [16]. Moreover, in a recent study, previous antibiotic exposure for more than 4 days is strongly associated with negative results on biopsy specimen culture [17]. On the other hand, in other studies, pathogen recovery in biopsy specimen culture is not significantly affected by previous antibiotic use $[7,12]$. In the present study, although it was examined in only one patient, neither the duration of previous antibiotic exposure nor pausing antibiotic use before obtaining blood and biopsy specimen cultures influenced the negative result of cultures, which appears inconsistent with the guideline recommendation. However, this might be an exceptional case involving Staphylococcus aureus, as reported in a recent study [18]. Although the inconsistent results should be further investigated, the present findings suggest that blood culture and, if possible, biopsy specimen culture should be performed regardless of previous antibiotic exposure. Since the sample numbers are small $(n=23)$, no statistical analysis was performed on any aspect of the data in the present study.

\section{Conclusion}

In conclusion, we first described the detailed characteristics of patients with PVO in a Japanese suburb. In an investigation of the causative pathogen of $\mathrm{PVO}$, the present study revealed that the combined use of blood culture and biopsy specimen culture could increase the detection rate, which might be helpful for selecting appropriate antibiotics and promptly initiating treatment.

\section{Conflicts of interest}

On behalf of all authors, the corresponding author declares that no conflict of interest exists.

\section{Ethical statement}

All procedures involving human participants in the present study were performed according to the ethical standards of the institutional and/or national research committee and in accordance with the 1964 Declaration of Helsinki and its later amendments or comparable ethical standards. In this article, no study involving animals was conducted by any of the authors.

\section{References}

1. Grammatico L, Baron S, Rusch E, Lepage B, Surer N, et al. (2008) Epidemiology of vertebral osteomyelitis (VO) in France: Analysis of hospital-discharge data 2002-2003. Epidemiol Infect 136: 653-660.

2. Gasbarrini AL, Bertoldi E, Mazzetti M, Fini L, Terzi S, et al. (2005) Clinical features, diagnostic and therapeutic approaches to haematogenous vertebral osteomyelitis. Eur Rev Med Pharmacol Sci 9: 53-66. 
Citation: Kamono M, Kabeya Y, Sohara E, Taoda A, Matsumoto T, Mine T, Haseb T, Ueda A, Takagi A and Higaki M (2017) The Etiology of Pyogenic Vertebral Osteomyelitis and Evaluation of Biopsy Specimen Cultures in Hospitalized Patients. J Med Microb Diagn 6: 261. doi:10.4172/2161-0703.1000261

Page 5 of 5

3. Mylona E, Samarkos M, Kakalou E, Fanourgiakis P, Skoutelis A (2009) Pyogenic vertebral osteomyelitis: A systematic review of clinical characteristics. Semin Arthritis Rheum 39: 10-17.

4. Matsumoto T, Mine T, Hayashi T, Kamono M, Taoda A, et al. (2017) CT fluoroscopy-guided transsacral intervertebral drainage for pyogenic spondylodiscitis at the lumbosacral junction. Cardiovasc Intervent Radiol 40: 125-129.

5. Gupta A, Kowalski TJ, Osmon DR, Enzler M, Steckelberg JM, et al. (2014) Long-term outcome of pyogenic vertebral osteomyelitis: A cohort study of 260 patients. Open Forum Infect Dis 1: 1-8.

6. Kim CJ, Kang SJ, Choe PG, Park WB, Jang HC, et al. (2015) Which tissues are best for microbiological diagnosis in patients with pyogenic vertebral osteomyelitis undergoing needle biopsy? Clin Microbiol Infect 21: 931-935.

7. Garg V, Kosmas C, Young PC, Togaru UK, Robbin MR (2014) Computed tomography-guided percutaneous biopsy for vertebral osteomyelitis: A department's experience. Neurosurg Focus 37: 1-8.

8. Berbari EF, Kanj SS, Kowalski TJ, Darouiche RO, Widmer AF, et al. (2015) 2015 Infectious Diseases Society of America (IDSA) Clinical practice guidelines for the diagnosis and treatment of native vertebral osteomyelitis in adults. Clin Infect Dis 61: e26-46.

9. Enoch DA, Cargill JS, Laing R, Herbert S, Corrah TW, et al. (2008) Value of CT-guided biopsy in the diagnosis of septic discitis. J Clin Pathol 61: 750-753.

10. Pupaibool J, Vasoo S, Erwin PJ, Murad MH, Berbari EF (2015) The utility of image-guided percutaneous needle aspiration biopsy for the diagnosis of spontaneous vertebral osteomyelitis: a systematic review and metaanalysis. Spine J 15: 122-131.

11. Rankine JJ, Barron DA, Robinson P, Millner PA, Dickson RA (2004) Therapeutic impact of percutaneous spinal biopsy in spinal infection. Postgrad Med J 80: 607-609.

12. Sehn JK, Gilula LA (2012) Percutaneous needle biopsy in diagnosis and identification of causative organisms in cases of suspected vertebral osteomyelitis. Eur J Radiol 81: 940-946.

13. Skaf GS, Kanafani ZA, Araj GF, Kanj SS (2010) Non-pyogenic infections of the spine. Int J Antimicrob Agents 36: 99-105.

14. Walter G, Vernier M, Pinelli PO, Million M, Coulange M, et al. (2014) Bone and joint infections due to anaerobic bacteria: An analysis of 61 cases and review of the literature. Eur J Clin Microbiol Infect Dis 33: 1355-1364.

15. Lew DP, Waldvogel FA (2004) Osteomyelitis. The Lancet 364: 369-379.

16. Trampuz A, Piper KE, Jacobson MJ, Hanssen AD, Unni KK, et al. (2007) Sonication of removed hip and knee prostheses for diagnosis of infection. N Engl J Med 357: 654-663.

17. Kim CJ, Song KH, Park WB, Kim ES, Park SW, et al. (2012) Microbiologically and clinically diagnosed vertebral osteomyelitis: Impact of prior antibiotic exposure. Antimicrob Agents Chemother 56: 2122-2124.

18. Corrah TW, Enoch DA, Aliyu SH, Lever AM (2011) Bacteraemia and subsequent vertebral osteomyelitis: A retrospective review of 125 patients. QJM 104: 201-207. 